\title{
Relationship between Spinal Hemangioblastoma Location and Age
}

\author{
Tatsuya Yasuda, Tomohiko Hasegawa, Yu Yamato, Sho Kobayashi, Daisuke Togawa, \\ Tomohiro Banno, Hideyuki Arima, Shin Oe, Yukihiro Matsuyama
}

Department of Orthopaedic Surgery, Hamamatsu University School of Medicine, Hamamatsu, Japan

\begin{abstract}
Study Design: Retrospective case series.
Purpose: To investigate the relationship between tumor location and clinical characteristics.

Overview of Literature: Hemangioblastoma is a rare disease that develops in the central nervous system. Magnetic resonance imaging (MRI) is useful to evaluate hemangioblastomas. Hemangioblastoma's location is designated as intramedullary, intramedullary+extramedullary, or extramedullary by MRI.

Methods: We analyzed 11 patients who underwent surgery for spinal hemangioblastoma. Using T1 contrast axial MRI data, the cases were divided into three groups (intramedullary, intramedullary+extramedullary, and extramedullary). Patient demographics, MRI findings, and preoperative neurological status were analyzed and compared for each group.

Results: The average age of patients with intramedullary, intramedullary+extramedullary, and extramedullary hemangioblastoma was $34.0,64.4$, and 67.5 years, respectively. Patients in the intramedullary hemangioblastoma group were younger than the other groups. Extramedullary cases had a smaller syrinx compared to the other groups.

Conclusions: Age may play an important role in the hemangioblastoma tumor location and the subsequent diagnosis by an MRI.
\end{abstract}

Keywords: Spinal hemangioblastoma; Tumor location; Magnetic resonance imaging; Age

\section{Introduction}

Hemangioblastomas are vascular tumors found throughout the central nervous system [1-3] including the cerebellum, spinal cord, spinal nerves, and brain stem $[4,5]$. Spinal hemangioblastoma is a rare disease that accounts for $2 \%-15 \%$ of all spinal cord neoplasms [6-8]. Spinal hemangioblastoma may occur sporadically $(70 \%-80 \%$ of cases), or as a component of von Hippel-Lindau (VHL) disease (20\%-30\% of cases) [8-10].

Magnetic resonance imaging (MRI) is used to diagnose spinal cord hemangioblastoma. Compared to the spinal cord, hemangioblastoma is usually hypointense to isointense on T1-weighted sequences and isointense to hyperintense on T2-weighted sequences. On T1-weighted MRIs with contrast enhancement, the tumors appear as bright, enhanced lesions.

Although spinal hemangioblastomas have benign features, such as those associated with a World Health Organization grade I classification, patients often have rapid symptom progression and are treated with surgery. Therefore, it is important to identify the tumor location using preoperative MRI to achieve total tumor resection and improve the surgical outcome. Imagama et al. [11]

Received Jul 8, 2015; Revised Aug 10, 2015; Accepted Aug 27, 2015

Corresponding author: Tatsuya Yasuda

Department of Orthopaedic Surgery, Hamamatsu University of Medicine,

1-20-1 Handayama, Higashi-ku, Hamamatsu 431-3192, Japan

Tel: +81-53-435-2299, Fax: +81-53-435-2296, E-mail: t.yasuda0820@gmail.com 
classified the location of spinal hemangioblastomas using MRI and determined the association between tumor location and Ki67 activity using pathological findings.

The purpose of this study was to investigate the association between patient demographics and spinal hemangioblastoma location.

\section{Materials and Methods}

The study design was a retrospective chart review. Informed patient consent was obtained. Eleven patients who underwent surgery for spinal hemangioblastoma in our department from 2010 to 2013 were included in the study. All patients were diagnosed using preoperative plain and contrast MRI. Preoperative neurological status was evaluated according to the functional scale described by McCormic et al. [12] Patients underwent surgical removal using the posterior approach under a microscope.

The patients were divided into three groups based on tumor location (intramedullary, intramedullary+ extramedullary, and extramedullary) according to the Imagama's report [11]. Location was determined by $\mathrm{T} 1$ contrast axial MRI findings.

From our analysis, intramedullary hemangioblastomas were intramedullary focal enhanced (focal type) (Fig. 1). Intramedullary+extramedullary hemangioblastomas had strong signals for both the extramedullary and intramedullary components (snowman sign) (Fig. 2). Extramedullary hemangioblastomas showed compression of the

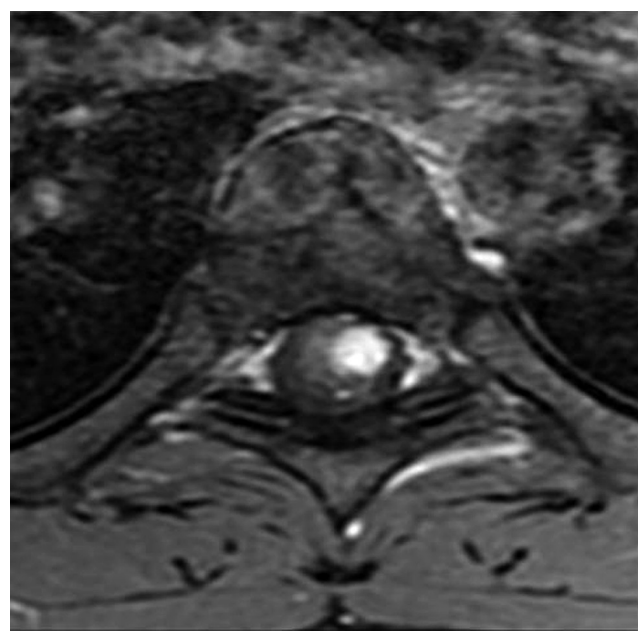

Fig. 1. A case of intramedullary hemangioblastoma. On the contrast axial T1WI magnetic resonance imaging, a focal tumor was observed in the intramedullary region (focal type). spinal cord from the outside, and the boundary lines between the tumor and the spinal cord were smooth (smooth type) (Fig. 3). Patient demographics, MRI findings, and preoperative neurological status were analyzed and compared between groups.

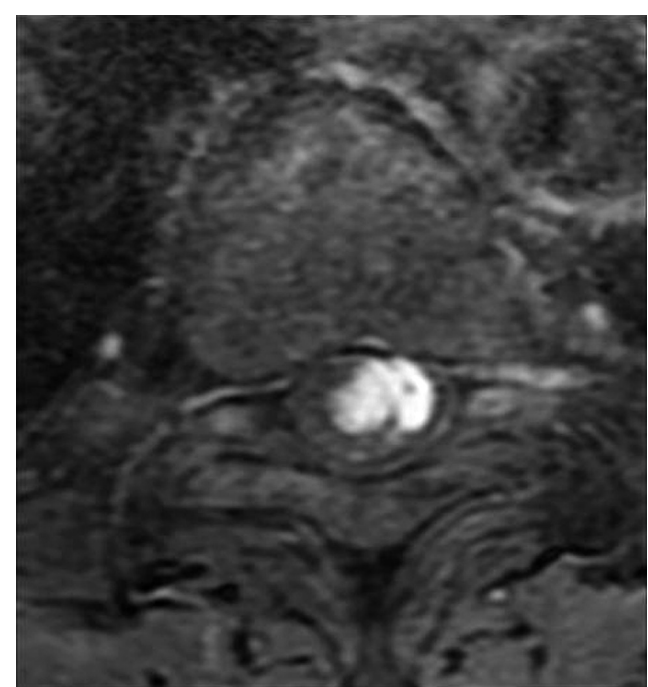

Fig. 2. A case of intramedullary+extramedullary hemangioblastoma. On the contrast axial T1WI magnetic resonance imaging, the enhanced tumor existed in intramedullary and extramedullary regions (snowman sign).

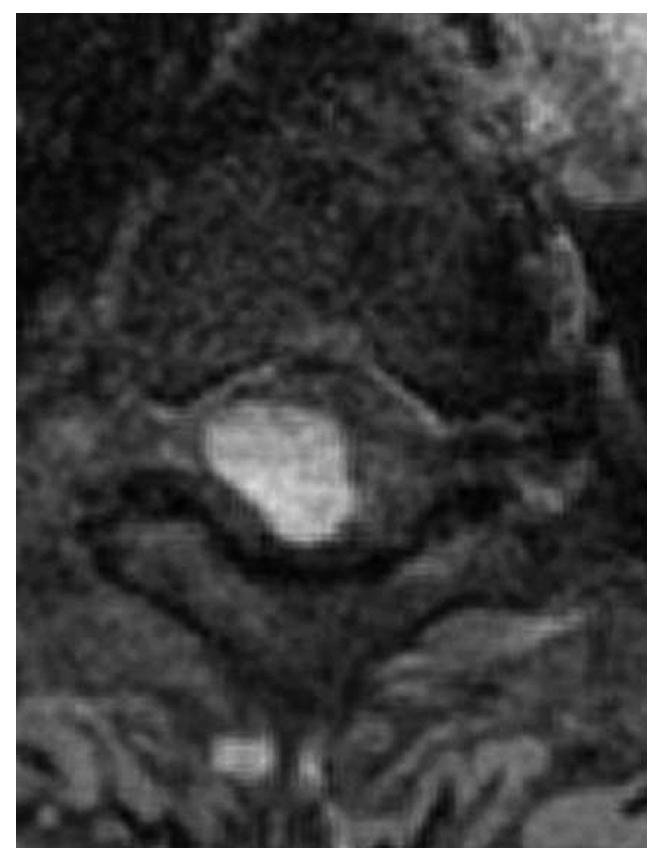

Fig. 3. A case of extramedullary hemangioblastoma. On the contrast axial T1WI magnetic resonance imaging, smooth boundary lines were clearly seen between the tumor and spinal cord. 
Table 1. Clinical and radiographic features of the 11 cases

\begin{tabular}{|c|c|c|c|c|c|}
\hline Location of tumor & Age (yr) & Sex & Level & Syrinx (vertebra) & McCormick grade \\
\hline \multicolumn{6}{|l|}{ Intramedullary (focal) } \\
\hline & 42 & Female & Cervical & 7 & I \\
\hline & 55 & Male & Cervical & 6 & I \\
\hline & 25 & Male & Thoracic & 10 & I \\
\hline & 14 & Male & Cervical & 15 & I \\
\hline Average & 34.0 & - & - & 9.5 & - \\
\hline \multicolumn{6}{|c|}{ Intra+extramedullary (snowman) } \\
\hline & 78 & Male & Thoracic & 14 & I \\
\hline & 64 & Female & Cervical & 12 & I \\
\hline & 62 & Male & Thoracic & 2 & $\|$ \\
\hline & 58 & Male & Cornus & 5 & I \\
\hline & 60 & Male & Thoracic & 11 & I \\
\hline Average & 64.4 & & - & 8.8 & - \\
\hline \multicolumn{6}{|l|}{ Extramedullary (smooth) } \\
\hline & 54 & Male & Cornus & 3 & I \\
\hline & 81 & Female & Thoracic & 4 & $\|$ \\
\hline Average & 67.5 & - & - & 3.5 & - \\
\hline
\end{tabular}

\section{Results}

Patient demographics and MRI findings are summarized in Table1.

\section{Patient characteristics and neurological status}

Eight males and 3 females were included in this study. The average age of these patients was 53.9 years (range, 14-81 years). One patient was diagnosed with VHL disease (Case 10). Nine patients were classified as McCormick grade I, and two patients were classified as McCormick grade II.

\section{MRI findings}

Four cases had tumors in the cervical spine, 5 cases in the thoracic spine, and 2 cases in conus region. In all cases, the presence and extent of syrinx ranged from 2 to 15 vertebrae (mean, 7.9 vertebrae) on the sagittal T2WI MRI. Intramedullary (focal type), intramedullary+extramedullary (snowman sign), and extramedullary (smooth type) tumors were present in 4, 5, and 2 cases, respectively.

The average age of patients with intramedullary, intramedullary+extramedullary, and extramedullary heman- gioblastoma was 34.0, 64.4, and 67.5 years, respectively. Patients with intramedullary hemangioblastoma were younger than the other groups. In extramedullary cases, the presence and extent of syrinx was 2 and 3 vertebrae, respectively. The average presence and extent of syrinx was 9.5 vertebrae and 8.8 vertebrae among intramedullary cases and intramedullary+extramedullary cases, respectively. Syrinx was smaller in extramedullary cases.

Preoperative neurological status was good, and no difference was observed between the three groups.

\section{Discussion}

Spinal hemangioblastoma is a relatively rare tumor. It predominately occurs in male patients, with a reported male/ female ratio ranging from $1 \cdot 6: 1$ to $5 \cdot 5: 1[2,6,10,13]$. On average, presentation occurs in the fourth decade of life.

Spinal hemangioblastomas occur as intramedullary tumors that progress from under the pia matter to an intramedullary lesion. Thus, intramedullary tumors are most common. Imagama et al. reported that $84.6 \%$ of hemangioblastomas were intramedullary, $11.5 \%$ were intramedullary+extramedullary, and $3.8 \%$ were extramedullary. In contrast, Deng et al. [14] reported that the 
percentage of tumors located in intramedullary, intramedullary+extramedullary, and extramedullary regions was $41 \%, 37 \%$, and $22 \%$, respectively. Our data were similar to those obtained by Deng et al. [14].

Previous case reports showed that the average age of patients with extramedullary hemangioblastoma was greater than the average age of patients with all types of hemangioblastomas, with most patients in their 50's (46-66 years) $[13,15-19]$. In our study, the patients with extramedullary hemangioblastoma were 54 and 81 years old. Thus, patients with extramedullary hemangioblastoma appear to be older than intramedullary cases in agreement with the previous reports.

Extramedullary hemangioblastoma causes no signal change in intramedullary regions, as shown in previous reports $[13,17,19]$. In this study, extramedullary hemangioblastoma cases had smaller syrinx compared to intramedullary and intramedullary+extramedullary cases. The syrinx findings on the sagittal MRI displays allow for differentiation between extramedullary, intramedullary, and intramedullary+extramedullary hemangioblastoma.

It is often difficult to differentiate hemangioblastomas from other intradural tumors, such as meningiomas by MRI. In particular, it is difficult to discriminate extramedullary hemangioblastoma and meningioma, because extramedullary hemangioblastoma have small syrinx. Age could be a factor used to diagnose hemangioblastoma.

The main limitation of this study was the small sample size. More cases are needed to increase the accuracy of these results. Because the number of hemangioblastoma cases is limited, a multicenter research study would be more useful than a single institute study. The second limitation is that the reason for the association between tumor location and patient age is unclear. Nevertheless, this study indicated that age might be an important factor to characterize hemangioblastoma.

\section{Conclusions}

The average age of patients with intramedullary hemangioblastoma was 34.0 years, which was lesser than those for patients with extramedullary and extramedullary+ intramedullary hemangioblastomas. Thus, age may play an important role in the likelihood of a hemangioblastoma location. Age should be considered when diagnosing hemangioblastoma by MRI.

\section{Conflict of Interest}

No potential conflict of interest relevant to this article was reported.

\section{References}

1. Lee DK, Choe WJ, Chung CK, Kim HJ. Spinal cord hemangioblastoma: surgical strategy and clinical outcome. J Neurooncol 2003;61:27-34.

2. Mandigo CE, Ogden AT, Angevine PD, McCormick PC. Operative management of spinal hemangioblastoma. Neurosurgery 2009;65:1166-77.

3. Na JH, Kim HS, Eoh W, Kim JH, Kim JS, Kim ES. Spinal cord hemangioblastoma: diagnosis and clinical outcome after surgical treatment. J Korean Neurosurg Soc 2007;42:436-40.

4. Glasker S, Berlis A, Pagenstecher A, Vougioukas VI, Van Velthoven V. Characterization of hemangioblastomas of spinal nerves. Neurosurgery 2005;56:503-9.

5. Kanno H, Yamamoto I, Nishikawa R, et al. Spinal cord hemangioblastomas in von Hippel-Lindau disease. Spinal Cord 2009;47:447-52.

6. Chu BC, Terae S, Hida K, Furukawa M, Abe S, Miyasaka K. MR findings in spinal hemangioblastoma: correlation with symptoms and with angiographic and surgical findings. AJNR Am J Neuroradiol 2001; 22:206-17.

7. Cristante L, Herrmann HD. Surgical management of intramedullary hemangioblastoma of the spinal cord. Acta Neurochir (Wien) 1999;141:333-9.

8. Mehta GU, Asthagiri AR, Bakhtian KD, Auh S, Oldfield EH, Lonser RR. Functional outcome after resection of spinal cord hemangioblastomas associated with von Hippel-Lindau disease. J Neurosurg Spine 2010;12:233-42.

9. Bostrom A, Hans FJ, Reinacher PC, et al. Intramedullary hemangioblastomas: timing of surgery, microsurgical technique and follow-up in 23 patients. Eur Spine J 2008;17:882-6.

10. Conway JE, Chou D, Clatterbuck RE, Brem H, Long DM, Rigamonti D. Hemangioblastomas of the central nervous system in von Hippel-Lindau syndrome and sporadic disease. Neurosurgery 2001;48:55-62.

11. Imagama $S$, Ito $Z$, Wakao $N$, et al. Differentiation of localization of spinal hemangioblastomas based on imaging and pathological findings. Eur Spine J 
2011;20:1377-84.

12. McCormick PC, Torres R, Post KD, Stein BM. Intramedullary ependymoma of the spinal cord. J Neurosurg 1990;72:523-32.

13. Barbosa-Silva E, Carvalho GT, Frota Mde O, Sousa AA, Souza CB. Intradural extramedullary hemangioblastoma. Arq Neuropsiquiatr 2009;67:530-3.

14. Deng X, Wang K, Wu L, et al. Intraspinal hemangioblastomas: analysis of 92 cases in a single institution: clinical article. J Neurosurg Spine 2014;21:260-9.

15. Wisoff HS, Suzuki Y, Llena JF, Fine DI. Extramedullary hemangioblastoma of the spinal cord: case report. J Neurosurg 1978;48:461-4.

16. Brisman JL, Borges LF, Ogilvy CS. Extramedullary hemangioblastoma of the conus medullaris. Acta
Neurochir (Wien) 2000;142:1059-62.

17. Taniguchi S, Ogikubo O, Nakamura T, et al. A rare case of extramedullary-intradural hemangioblastoma in the thoracic spine. Spine (Phila Pa 1976). 2009;34: E969-72.

18. Kitanaka C, Kuwahara M, Teraoka A. Intradural, purely extramedullary hemangioblastoma of the spinal cord: case report. Neurol Med Chir (Tokyo) 1993; 33:377-80.

19. Toyoda H, Seki M, Nakamura H, Inoue Y, Yamano Y, Takaoka K. Intradural extramedullary hemangioblastoma differentiated by MR images in the cervical spine: a case report and review of the literature. J Spinal Disord Tech 2004;17:343-7. 